\title{
Product Mix Planning in Semiconductor Manufacturing
}

\author{
I-Hsuan Hong \\ National Taiwan University \\ Taipei, Taiwan, R.O.C.
}

\author{
$\mathrm{Y}-\mathrm{C}$ Chou \\ National Taiwan University \\ Taipei, Taiwan, R.O.C. \\ ychon(a)ccms.ntu.edu.tw
}

\author{
C.Y Kuo, L-C. Lu \\ UICC \\ HsinChu, Taiwan, R.O.C.
}

\begin{abstract}
To cope with product demand and price fluctuations and rapid technology transition, many semiconductor manufacturers have broadened their product scope to wider ranges of inemory and logic devices in recent years. Product mix planning emerges as a critical business capability to exploit market opportunities. This paper focuses on product mix planning in a foundry manufacturing environment. Issues discussed include levels of planning granularity, mix planning and bottleneck-based analysis. A software system has been implemented that provides both product mix solutions and sensitivity information of product price and tool capacity. Used as a planning tool, the system will improve fab agility under dynamic market conditions.
\end{abstract}

\section{INTRODUCTION}

With a given level of fab capacity, the task of product mix planning is to determine a portfolio of product types and production volumes. Traditionally, product mix planning involves two issues: cost accounting of capacity at the process routing level [2] and combinatorial optimization of product mixes. The objective of cost accounting is to accurately cstimate the manufacturing cost of each product type, whereas mix optimization seeks to maximize the efficiency of capacity allocation among products. Typical methods for mix optimization are integer linear programming and the theory of constraint [1]. It has been shown by numerical examples that both methods are actually equivalent and could lead to the same solutions [3].

Semiconductor manufacturing industry is very dynamic in process and tool technology, product demand and fab capacity expansion, giving rise to a number of unique phenomena to product mix planning. First, process flow is long; the flow time is usually more than one month. Even if a large time bucket, such as month, is used in planning, wafer lots released to the fab in onc time period will introduce workloads to other time periods, resulting in inaccuracy of workload calculation. Second, fabs usually go through continuing phases of expansion and process improvement; tool portfolio and capacity may not be in any steady state. In addition, wafer fabs are large, complex operation systems of strong queuing network behavior. Product mix planning involves performance evaluation and trade-off between throughput, utilization and cycle time.

The remainder of the paper is organized as follows. An analysis of the problem is presented in Section 2. In Section 3 , the effect of time granularity on planning accuracy is analyzed to identify the best time bucket for product mix planning. In Section 4, mixed integer linear programming models for multiple, heterogeneous classes of products are outlined, A procedure based on botnleneck analysis is described in Section 5. Numerical cases are presented in Section 6, covering the necessity of cycle time uffset. objective functions, and bottleneck-based planning.

\section{PROBLLM ANALYSIS}

Four classes of product demands are identifiable for foundry fabs: committed orders, fixed makc-to-order demand, variable make-to-order demand and make-to-stock orders (Table 1). Committed orders are inherited from previous planning periods. Fab capacity must be reserved for committed orders as a matter of course. Make-to-order demands are fixed in price, but the volume could be either fixed according to contracted delivery terms or be flexible allowing mancuvering room for operation efficicncy. Make-to-stock items are standard products. They arc usually used to fill up capacity.

\begin{tabular}{|c|c|c|c|}
\hline Class & Order type & Volume & Price \\
\hline \hline$O_{1}$ & Committed orders & Fixed & Fixed \\
$O_{2}$ & Make-10-order & Fixed & Fixed \\
$O_{3}$ & Make-to-order & Range & Fixed \\
$O_{4}$ & Make-to-stock & Variable & Variable \\
\hline
\end{tabular}

Table 1. Product classes.

Product mix planning requires a sound method for calculating workload and capacity. There are already several implementations of static capacity models in the literature that take into consideration cycle time offset, yield and efficiency $[4,5]$. The computation logic is straightforward but there is still a question of tradeoff between precision and computation time. Common industry practice for capacity planning is to use month as the time bucket and workload is calculated based on monthly demand data.

In this sudy, candidate orders are called denand batches, which are characterized by product type (i) and due time $(t)$ attributes. A static capacity model is used 10 calculate the workload primitives $\left(w_{b, j, k t}\right)$ that is due to batch $b$ and process step $j$ for tool $k$ in time $t$ taking into consideration lead-time offsct of each step. Next, workload. primitives are summed up to generate unit workload $\left(W_{b, k, 1}\right)$ for each unit of product. The time $t$ of both workload primitives and unit workloads is expressed in day. Unit workloads in the same aggregate periods are then combined 
to generate aggregate workload $W_{b, k, p}$ for time period $p$. There are two parameters in the calculation. One is the number of work releases for each demand batch and the other is the time bucket for aggregate period $(p)$. The time bucket could be month, week or other time units. The computation time would be less if a large time bucket is used. but a small time bucket will allow bottleneck tools to be better pinpointed. Similarly, if daily work rclease were modeled, mimicking fab operation, workload calculation would be more accurate at the expense of more computation time. Thus, there is an issue of determining the time bucket size and work release frequency for workload calculation.

Performance evaluation of fab operation is a complex issue. A product mix plan may not be realizable under dynamic fab environment. Therefore, the robustness of product mix is an important concern. As the output is constrained by shifting bottlenecks, the variation of workload is a surrogate measure of robustness.

\section{PRECISION WORKLOAD CALCULATION}

To determine the best granularity level for product mix planning, four levels of time bucket size and four levels of work release frequency are paired and the workload variation of the sixteen cases is compared. I ct $\mathrm{G}-1, \mathrm{G}-2, \mathrm{G}$ 4 , and G-30 represent time bucket size of month, two-weck, weck and day, respectively, and let F-1, F-2, F-4 and F-30 represent work release frequency of monthly, biweekly, weekly and daily. For stationary product mix, total workloads for toolsets should be stationary. That is to say the mean of workload for each toolset is the same for all cases. However, due to mismatch in work release and time bucket size, the variation of workload is not the same. This phenomenon is illustrated in Figure 1.

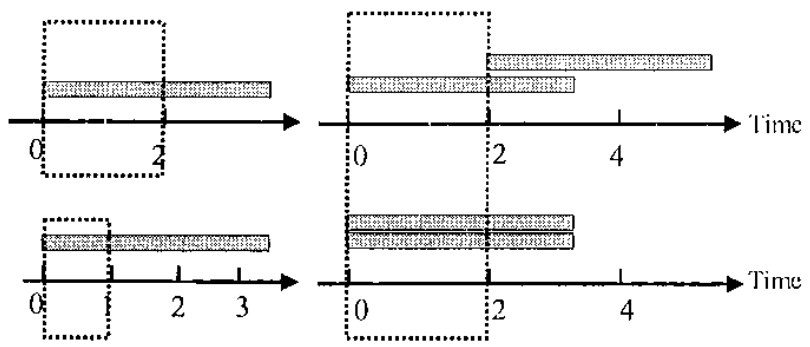

Figure 1. Effect of granularity on workload variation.

Numerical calculation of workioad has been done for a product mix over a planning horizon of one year. The squared coefficient of variation (SCV) is chosen to be the measure of workload variability (Equation 1) and Table 2 summarizes the average SCV of workload. Let $W_{k, p}=\Sigma_{b}, W_{b, k, p}$.

$$
\begin{aligned}
& S C V_{k}=\frac{V\left[W_{k, p}\right]}{\left(E\left[W_{k, p}\right]\right)^{2}} \quad \forall k \\
& \text { Average } S C V \text { of workload }=E\left[S C V_{k}\right]
\end{aligned}
$$

For each granularity level, more accurate estimation of workload distribution can not be gained by adopting a higher release frequency and additional noise will be introduced by adopting a lower release frequency. It can he concluded that work release frequency should be consisten with the time bucket in granularity.

\begin{tabular}{|c|c|c|c|c|}
\hline & $F^{-}-1$ & $F-2$ & $F-4$ & $F-30$ \\
\hline$G-1$ & 0 & 0 & 0 & 0 \\
\hline$G-2$ & 0.139 & 0 & 0 & 0 \\
\hline$G-4$ & 0.416 & 0.154 & 0 & 0 \\
\hline$G-30$ & 4.645 & 2.162 & 1.095 & 0 \\
\hline
\end{tabular}

Table 2. Workload variation of granularity levels.

In the first set of computation, work relcase follows a prese interval and each month is assumed to have thirty days. $\wedge$ second set of computation has been done to compare the robustness of G1-Fi, G2-H2, G4-F4 and (330-130. (The four cases represent four aggregate schemes for the time period $p$.) Table 3 summarizes the experiment setting. The calendar months are used. $\Lambda$ month may have 28,30 or 31 days. This also affects the inter-release time between work releases. For instance, the time between two work releases may be 14,15 , or 16 days for the case of (i2-12, depending on the month. The SCV of released work can be computed from the inter-release time.

SCV of released work $=\frac{V[\text { interelease time }]}{(E[\text { interrelease time }])^{2}}$

\begin{tabular}{|c|c|c|c|c|}
\hline Experiment & $\begin{array}{c}\text { Work release } \\
\text { frequency } \\
\text { (per month) }\end{array}$ & $\begin{array}{c}\text { Inter-release time } \\
\text { of work } \\
\text { (days) }\end{array}$ & $\begin{array}{c}\text { SCV of } \\
\text { relcased } \\
\text { work }\end{array}$ & $\begin{array}{c}\text { Time } \\
\text { period } \\
\text { bucket }\end{array}$ \\
\hline G1-F1 & 1 & 28,30 or 31 & 0.0009 & Month \\
\hline G2-F2 & 2 & 14,15 or 16 & 0.0015 & Bi-week \\
\hline$G 4-\sqrt{4} 4$ & 4 & 7 or 8 & 0.0042 & Week \\
\hline G30-F30 & 28,30 or 31 & 1 & 0.0 & Daly \\
\hline
\end{tabular}

Table 3. The setting of robustness experiments.

Let $X$ be a random variable for total workload. The computed SCV is modified by theoretical SCV to normalize the difference in workload aggregation. A fidelity measure. defined as the ratio between the modified workload SCV and the SCV of release work, is used to compare the robustness of the four granularity cases:

$$
\text { fidelity }=\frac{\text { computed workload SCV }}{S C V \text { of released work theoretical workload SCV' }}
$$

A large value of fidelity means that the vartation in released work is magnified and shows up in observed variation. The results are summarized in Table 4 . The fidelity ratio of G1-F1 and G2-F2 is comparable, and so is that for G4-F4 and G30-F30. However, G4-F4 and G30F30 require higher computation load compared with GI-Fl and G2-F2. It is concluded that cither week or month should be used as the time bucket size. 


\begin{tabular}{|l||c|c|c|c|}
\hline \multicolumn{1}{|c|}{ Experiment } & $\mathrm{G} 1-\mathrm{F} 1$ & $\mathrm{G} 2-\mathrm{F} 2$ & $\mathrm{G} 4-\mathrm{F} 4$ & $\mathrm{G} 30-\mathrm{F} 30$ \\
\hline $\begin{array}{l}\text { Aggregate } \\
\text { relcased load }\end{array}$ & $\sum_{30} X_{i}$ & $\sum_{14} X_{i}$ & $\sum_{7} X_{i}$ & $X_{i}$ \\
\hline $\begin{array}{l}\text { Theoretical } \\
\text { workload SCV }\end{array}$ & $\frac{\sigma^{2}}{30 \mu^{2}}$ & $\frac{\sigma^{2}}{14 \mu^{2}}$ & $\frac{\sigma^{2}}{7 \mu^{2}}$ & $\frac{\sigma^{2}}{\mu^{2}}$ \\
\hline workload SCV & 0.0041 & 0.0146 & 0.0144 & 0 \\
\hline Fidelity ratio & 4.6795 & 4.5541 & 0.7954 & 0 \\
\hline
\end{tabular}

Table 4 . Robustness analysis.

\section{PRODUCT MIX OPTIMIZATION}

Several objectives are of interest in product mix optimization: to maximize profit, to maximize wafer output, to maximize tool utilization or to maximize a hybrid model of profit and utilization. A mixed integer linear program (MIP) is used to find optimal product mix and mix ratio (i.e., type and volume). The decision variables, parameters and constraints are as follows.

\section{Decision variables:}

$X F_{b,}: \quad 0-1$ variable for $b \in O_{2} ; X_{1},=1$ if batch $b$ is selected, $X_{b},=0$ otherwise

$X R_{b}$ : Quantity of batch $b, b \in \mathrm{O}_{3}$

$X V_{b,}$ : The number of lots of batch $b, b \in O$,

$S_{k t}: \quad$ Residual capacity on tool $k$ in period $t$

$R_{m k t}$ : Fraction of tool $m$ to back up tool $k$ in period $t$

\section{Parameters:}

$C M_{i}$ : Profit margin of product type $i$ of $\mathrm{O}_{2}$ and $\mathrm{O}_{3}$

$C M_{i t}$ : Profit margin of product type $i$ of $\mathrm{O}_{4}$ in period $t$

$Q_{b}: \quad$ Quantity of batch $b$ for $\mathrm{O}_{2}$ orders

$Q_{b}^{\prime \prime}$ : Maximal quantity of batch $b$ for $O_{3}$ orders

$Q_{b}^{\prime}$ : Minimal quantity of batch $b$ for $O_{3}$ orders

$r_{m k}$ : Backup efficiency of tool $m$ with respect to tool $k$

$C P_{k t}$ : Capacity of tool $k$ in time period $t$

$\delta: \quad$ The range parameter (\%) of production volume

\section{Maximizing profit:}

$\operatorname{Max} \sum_{b \in O_{2}} C M_{i(b)} \cdot Q_{b} \cdot X F_{b}+\sum_{b \in O_{3}} C M_{i(b)} \cdot X R_{b}+\sum_{b \in O_{4}} C M_{i(b), q(b)} \cdot X V_{b}$

Maximizing wafer output:

$\operatorname{Max} \sum_{b \in O_{2}} Q_{b} \cdot X F_{b}+\sum_{b \in O_{3}} X R_{b}+\sum_{b \in O_{4}} X V_{b}$

Maximizing tool utilization:

$\operatorname{Min} \sum_{i} \sum_{k} S_{k t}$

Hybrid model to maximize profit and utilization:

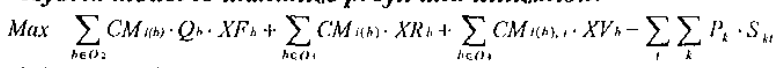

The penalty $\left(P_{k}\right)$ for residual capacity is equal to the weighted unit profit multiplied by throughput of tool. Let $W_{b k t}$ be the workload contributed by one product unit of batch $b$. The constraints for capacity, bounds on volume, and incremental change in volume are as follows.

$$
\begin{gathered}
\sum_{b \in O_{2}} X F_{b} \cdot Q_{b} \cdot W_{b k t}+\left(\begin{array}{cc}
\left.\sum\left(X R_{b}+X V_{b}\right) \cdot W_{b k l}\right)+S_{k t}=C P_{k t} \quad \forall k, t \\
\left.Q_{b}^{l} \leq X O_{3} \cup O_{4}\right)
\end{array}\right. \\
(1-\delta) X R_{l} \leq X R_{b} \leq(1+\delta) X Q_{l} \quad \forall b \in O_{3} \quad \forall b \in O_{3} \\
(1-\delta) X V_{l} \leq X V_{b} \leq(1+\delta) X V_{l} \quad \forall b \in O_{4}
\end{gathered}
$$

where $t(b):=t(l)+1$ and the production quantity for a period is within the $(1 \pm \delta)$ range of that in previous period.

Let $G(k)$ be the set of backup tools for tool $k$ and $I /(k)$ be the tools that are backed up by tool $k$. The capacity and workload reassignment constraints are:

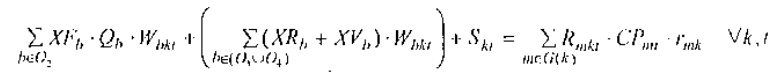

$$
\begin{aligned}
& \sum R_{n k t}=1 \quad \forall m, t \\
& k \in \|(m)
\end{aligned}
$$

The fraction $\left(R_{m k t}\right)$ is a $0-1$ variable if fractional backup is not allowed in a time period, otherwise it is a real number.

These MIP formulations provide shadow price of 100 ! capacity and marginal profit reçuirement for products (Table 5). The shadow price of a tool is the marginal rate of revenue over tool capacity. The marginal profit requirement for a product is the required increment in unit profit for a product to be selected for production.

\begin{tabular}{cccccccc}
\hline Tool Period & $\begin{array}{c}\text { Shadow } \\
\text { Price }\end{array}$ & Product & Period & Volume & $\begin{array}{c}\text { Marginal Profit } \\
\text { Requirement }\end{array}$ \\
\hline 48 & 3 & 5.38 & & 2 & 3 & 0 & 0.110 \\
53 & 3 & 0.82 & 2 & 4 & 0 & 0.027 \\
$:$ & $:$ & $\vdots$ & $:$ & $:$ & $\vdots$ & $:$ \\
10 & 13 & 1.40 & 3 & 3 & 0 & 0.197 \\
$:$ & $:$ & $:$ & $:$ & $:$ & $:$ & $:$
\end{tabular}

Table 5. Shadow price and marginal profit requirement.

The sensitivity analysis also covers unit profit allowence and capacity allowance (Table 6). 'l'he optimal mix ratio

\begin{tabular}{|c|c|c|c|c|c|}
\hline Tool & Period & Allowance & l'roduct & Peries & Alluwatlec \\
\hline 1 & 3 & $(220.7$, infinity) & 1 & 3 & $(0.89,1,3(j)$ \\
\hline 1 & 4 & (186.2, inlinity) & 1 & 4 & (1) $7 \%, 3.3(1)$ \\
\hline : & : & : & . & & \\
\hline 61 & 15 & (105.7, inlinity) & 2 & 0 & (10) 4.113 \\
\hline : & : & & & : & \\
\hline
\end{tabular}
will remain the same when the unit profit of a product fluctuates within its profit allowance. The capacity allowance of a single tool delimits the range of tool capacity within which the optimal product mix will remain the same.

Table 6. Capacity and unit profit allowances.

\section{BOTTLENECK-BASED PLANNING}

Since in general only a few constraints are binding and it is binding constraints that dominate the solution, nonbottleneck tools could be excluded to reduce the formulation size. Howcver, the bottleneck tends to shift when product mix changes from period to period. Bottleneck determination and product mix are convolute decisions. An iterative procedure has been used to identily the bottleneck tool set. A reduced cotmulation covering a part of the planning horizon is first used to determine an initial product mix. The bottleneck tool set is nexl determined from the product mix. $\Lambda$ formulation based on the bottleneck tool set is in turn used for the entire planning 
horizon to optimize product mix. Product mix and bottleneck tools are revised iteratively. The procedure is:

(1) Select a reduced time span, which should be longer than cycle time, at the center of the specified planning horizon.

(2) Solve an MIP formulation for time periods in the reduced time span.

(3) Identify bottleneck tools based on a utilization threshold.

(4) Solve an MIP formulation on bottleneck tools for all periods.

(5) Check the workload of tools. If there are overloaded tools, add them to the bottleneck tool set and go to Step (4), otherwise, stop.

The setting of bottleneck threshold affects the efficiency of the procedure. If a lower value is used, more tools will be deemed bottleneck, some crroneously. If a high value is used, initially few tools will be included in the bottleneck set. But, as more time periods are included in the formulation in later iterations, new bottleneck tools will surface. Figure 2 shows a relation between utilization threshold and the size of the bottleneck tool set. When the threshold is set at $0.5,65$ toolsets are included in the bottleneck set. No additional bottleneck tools surfaced afterward. When the threshold is set at $0.95,15$ tools are initially included in the set. But in a sccond iteration, 40 additional tools surfaced as bottleneck tools, bringing the total bottleneck tool count to 55. Figure 2 shows that a threshold between 0.87 to 0.92 results in the snallest bottleneck set ( 39 out of 116 toolscts).

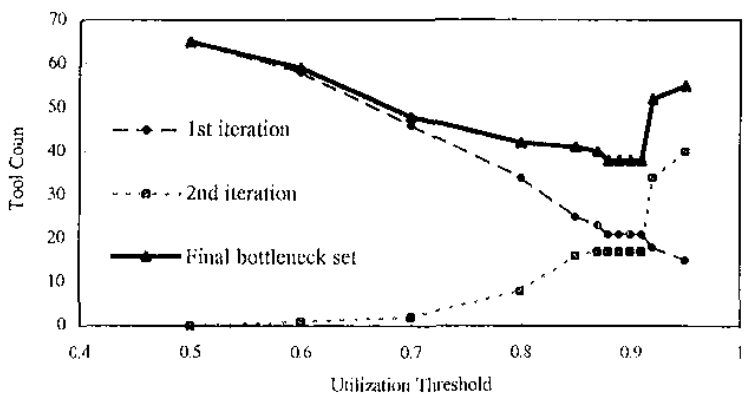

Figure 2. Determination of the bottleneck tool set.

\section{NUMERICAL RESULTS}

The above methodology has been implemented and run on industry data. The workload calculation logic described in Section 3 includes cycle time offset. This meticulous detail of calculation is necessary. Two product mix solutions were computed, one with cycle time offset and the other with zero cycle time. The planning horizon is from period 61 to period 105. Table 7 shows the resulted profit and output. Although the output and profit are higher if cycle time offset is not included, that results are actually infeasible as tool capacity is violated at 122 tool-periods in follow-up checking.

\begin{tabular}{|c|c|c|c|}
\hline $\begin{array}{c}\text { Cycle time } \\
\text { offset }\end{array}$ & Total profit & Wafer output & $\begin{array}{c}\text { Overloaded } \\
\text { tool-period }\end{array}$ \\
\hline not included & 1,163 & 132,238 & $\frac{122}{122}$ \\
\hline included & 1,132 & 127,053 & Nonc \\
\hline
\end{tabular}

Table 7 . Effect of including cycle time offset.

The planning horizon is from period 61 to 79 , which can be solved using the regular MP formulation. The outcomes of the regular model and the bottleneck-based model are shown in Figure 3. The results are identical.
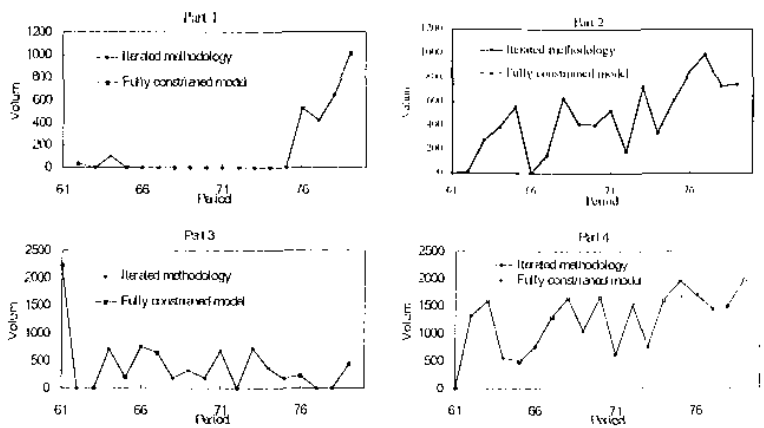

Figure 3. Results of bottleneck-based planning

\section{CONCIUSIONS}

This paper presents a methodology for product mix planning. It is shown that month or week, instead of day or two-week, should be used as the time buckel size for workload and product mix calculation. In additron, work release frequency should be at the same gramulanty level as time bucket size. Mixed integer linear programming formulations have been used to optimize product mix. When the problem size is large, a bottleneck-based procedure has been used. Finally, it is also show'n that it is essential to include cycle time offset calculation in product mix planning.

Acknowledgements: This study has been partially funded by NSC $(2212,2213,2622)$ of R.O.C. and UICC.

\section{RFFERENCES}

[1] E. M. Goldratt. The Haystack Syndrome. Croton-onHudson, NY: North River Press, 1990.

[2] J. G. Helmkamp. Managerial Accounting. John Wiley \& Sons, Inc, 1st ed., 1987, pp. 236-237.

[3] R. Iuebbe and B. Finch, "Theory of constraints and linear programming: a comparison." International Journal of Production Research, vol. 30, pp. 1471$1478,1992$.

[4] Jim Neudorff, "Static capacity analysis using Microsoft Visual Basic," International Conference on Semiconductor Manufacturing Operational Modeling and Simulation, 1999, pp. 207-212.

[5] W.-F. Wu, J.-I. Yang and J.-T. Liao, "Static capacity checking system with cycle time considered," The $7^{\text {th }}$ International Symposium on Semiconductor Manufacturing, 1998, pp. 307-310. 